\title{
Influence of global postural reeducation method on respiratory muscle strength and parkinsonian quality of life
}

\author{
Larissa Salgado de Oliveira Rocha', Larissa Natsumi Hosoda Mineshita², Luciane Lobato Sobral 2,3, \\ Lizandra Dias Magno², Marcio Clementino de Souza Santos², Rodrigo Santiago Barbosa Rocha²
}

\begin{abstract}
Background: Degenerative diseases such as Parkinson's disease can lead to postural changes and muscular strength, this generates greater impact on the individual, on his functional capacity, respiratory system, mobility and, quality of life (QOL). Objective: The aim of this study was to verify the influence of the Global Posture Reeducation (GPR) method on respiratory muscle strength and QOL in patients with Parkinson's disease. Methods: Twenty volunteers, the average of age is $48.8 \pm 6.22$ years, they are diagnosed with Parkinson's disease at level 2 to 3 by the Hoehn \& Yahr Scale. They were submitted to the protocol of treatment with the GPR method in the postures of Frog in the ground and ballerina, performed regularly twice a week lasting 60 minutes each session, for 6 weeks, totaling 12 sessions, evaluated by PDQ-39 quality of life questionnaire and by inspiratory pressure measurements and Maximum expiratory value. Results: In the quantitative analysis of inspiratory and expiratory muscle pressure, there was an increase in post-treatment for maximal inspiratory pressure (MIP) $(p<0.05)$ when compared to pre-treatment, as well as when compared with predicted values MIP and maximum expiratory pressure (MEP) presented higher values $(p<0.05)$. In terms of QoL domains: mobility $(p=0.0009)$, daily life activity $(p=0.0006)$, emotional well-being $(p=0.001)$, cognition $(p=0.01)$ and physical discomfort were statistically significant in post-treatment. Conclusion: The use of GPR in Parkinson's disease has shown to be effective in the treatment of respiratory muscle strength and QoL.
\end{abstract}

Keywords: Parkinson's Disease, Rehabilitation, Muscle Strength, Quality of Life.

\section{INTRODUCTION}

The process of human aging is accompanied by progressive physiological changes and decline of all biological systems, as well as the central nervous system (CNS). It suffers a decrease in brain volume due to progressive degeneration induced by senescence ${ }^{(1)}$, sometimes correlated with the onset of pathologies associated with the neurodegenerative process, generating considerable impacts on functional capacity ${ }^{(2)}$. In this context, one of the main pathologies inherent to this phase is Parkinson's disease (PD) is characterized by dysfunctions in the nuclei of the base, consequent to the degeneration of component neurons, of the nigrostriatal dopaminergic pathway ${ }^{(3)}$. Such depletion generates cognitive and motor disabilities, they are manifested by a classical tetrad, including resting tremor, bradykinesia, postural instability and muscular rigidity ${ }^{(4)}$.

The respiratory system is compromised by tetrad, leading to dysfunction due to respiratory muscle weakness in the diaphragm, pectoral and intercostal, and consequently, upper airway obstruction, atelectasis, retention of pulmonary secretions and infections, decreased pulmonary compliance, among others. The expiratory musculature is usually the most compromised, aggravating the disease by reducing the maximum expiratory pressure, lower expiratory flow and increase in residual volume. Thus, the main cause of decreased functional capacity and mortality of parkinsonian patients is related to respiratory dynamics ${ }^{(5)}$.

Therefore, the physiotherapeutic treatment of the PD patient should prioritize the delay of the biomechanical and functional alterations, through methods such as global postural reeducation (GPR), it treats the disharmonies of the human body taking into account the individual needs of each patient ${ }^{(6)}$. GPR is based on the benefits gained from shortened musculature and isometric contractions, as well as proprioceptive stimuli to promote stability, balance, and alignment of body segments, improvement of respiratory function, functionality, flexibility and quality of life $(\mathrm{Q} O \mathrm{~L})^{(7,8)}$.

The benefits of GPR are obtained from practical exercises avoiding and relieving several types of pain with movements that aid in stretching and in the shape of the muscles ${ }^{(9)}$. In view of the context of PD, the principles contained in the

Corresponding author: Rodrigo Santiago Barbosa Rocha Tv. Perebebuí, n. 2623, Marco, Belém-PA, Department of Human Movement Sciences, University of the State of Pará (UEPA), Belém (PA) Tel: +55 91 99234-0234, email: fisiorocha2000@yahoo.com.br

2 Universidade do Estado do Pará (UEPA), Belém (PA), Brazil.

Full list of author information is available at the end of the article.

Financial support: nothing to declare

Submission date 25 July 2017; Acceptance date 14 November 2017; Data de publicação 28 December 2017 
GPR implementation and the reduced number of studies, correlating the method as a tool for the rehabilitation of neurological disorders, especially PD, this study aimed to discuss the performance of physiotherapy through this method in the alterations in the respiratory muscle strength of these patients.

\section{METHODS}

The study was carried out at the Clinical School of Physiotherapy of the University of the Amazon after approval of the Ethics Committee of the Institution whose protocol $\mathrm{n}$ - 11490812.3.0000.5173 and signing of the free and informed consent form. Twenty volunteers of both sexes, aged between 45 and 60 participated in this study, they have idiopathic PD with grade 2 to 3 in the Modified Scale of Hoehn and Yahr ${ }^{(10)}$, they do not have cardiac arrhythmias and other associated pathologies. Exclusion criteria were considered cognitive deficit assessed by the mini mental state examination ${ }^{(11)}$, in case, they had presented during the treatment period the disease progression or presented unforeseen events such as falls and other types of domestic accidents that made impossible to attend the sessions, as well as those who were currently undergoing physical therapy. The flowchart of the volunteers in the study is shown in figure 1.

All volunteers were submitted to the evaluation of maximal respiratory pressures, they were positioned in a chair in a sitting position. A nasal clip was used and requested after spontaneous breathing cycles, an expiration followed by maximal forced inspiration to measure the momentary capacity of the inspiratory musculature to measure the expiratory musculature, an inspiration followed by a forced maximum expiration, lasting from 3 to 5 seconds, being considered 3 measures reproducible or acceptable and the average among the repetitions ${ }^{(12)}$. Maximal inspiratory pressure (MIP) was measured during effort initiated from the residual volume, while the maximal expiratory pressure (MEP) was measured from the total lung capacity. The results were compared in pre and post-treatment in the GPR group and related to predicted values of normality and with the Parkinson's group (PG), however, they did not obtain intervention.

The PDQ-39 Scale was used to evaluate the QoL, it was consisted of 39 items distributed in eight subscales: mobility, activities of daily living (ADL's), emotional well-being, stigma, social support, cognition, communication and physical discomfort, the score of each item can vary from 0 (never) to 4 (always or impossible for me), totaling a score ranging from 0 (no problem) to 100 (maximum problem level). The best score obtained by the individual is the perception of $\mathrm{QoL}^{(13)}$. Before long, the volunteers of the GPR group were submitted to the GPR sessions individually, twice a week with approximate duration of 60 minutes each session, totaling 12 sessions, for six weeks, being applied by one of the researchers has the training in the GPR method. The treatment was carried

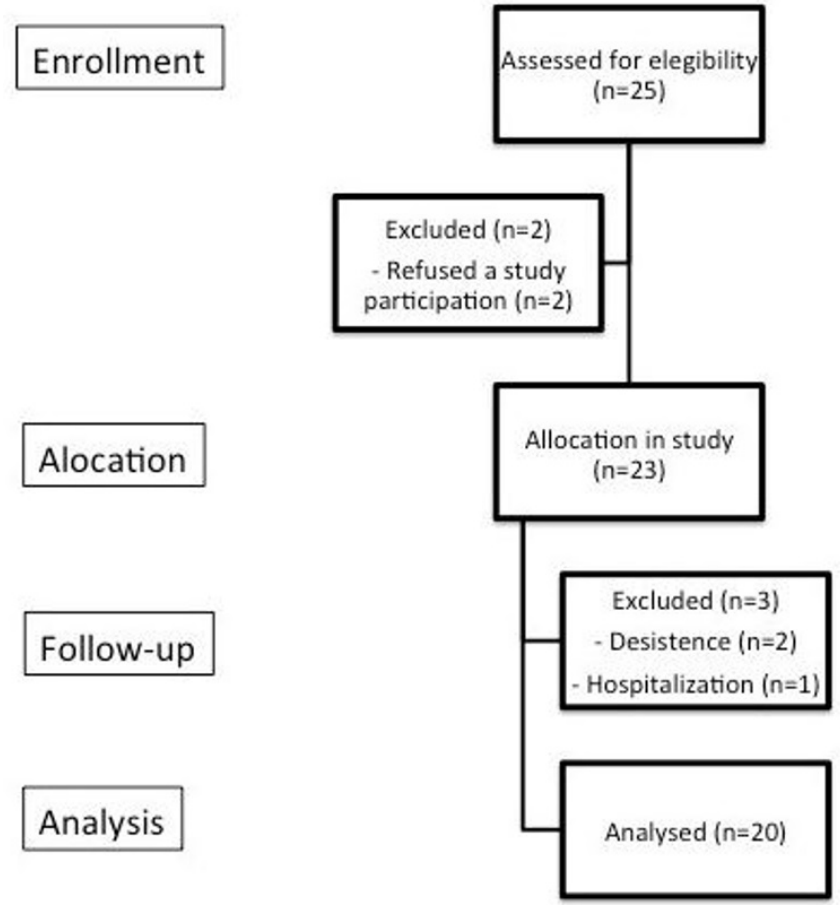

Figure 1. Flowchart of the volunteers.

out on a stretcher specific for the method, with dimensions of $2 \mathrm{~m}$ in length, $1 \mathrm{~m}$ in width and $75 \mathrm{~cm}$ in height and the postures chosen for application of the GPR were the ground frog and ballerina postures, they were maintained throughout cervical traction and paradoxical breathing. Breathing was taught to each volunteer before beginning the session and remembered in subsequent sessions, afterwards, it was started the application learning of each posture.

The frog posture was performed on the ground, where initially each volunteer was positioned in dorsal decubitus with open hip angle and arms open on the stretcher own GPR and with the arms of the same being kept open. The initial posture was maintained with arms in abduction, forearms in supination, the palms of the hands were facing upwards; lower limbs with abduction, hip flexion, and flexed knees until the entire toe of the soles of the feet. The dorsal pompage was performed, aiming the alignment of the dorsal and cervical curvatures of the vertebral column, while the sacral pompage sought the rectification of the lumbar spine. As the evolution of the posture occurred and with the therapist maintaining constant cervical traction and paradoxical breathing, the volunteer arms and the upper limb supported on the same was approximate of the trunk and the posture was finished as soon as the volunteer acquired global extension in decubitus dorsal to the anterior muscle chain ${ }^{(14)}$. Subsequently, the dancer's posture was adopted with the volunteer in standing position and initially remaining in semi-flexion of knees and hip. With the gradual advancement of the posture and the therapist maintaining constant cervical traction with the aid 
of paradoxical breathing, the volunteer finished the posture in standing position with extension of knees, hip and shoulders and flexion of trunk to posterior muscular chain ${ }^{(14)}$. During both postures, isometric contractions were also performed between the upper and lower segments and were variable among the volunteers, due to fatigue and muscle shortening presented by them. The isometric contractions were performed without joint movement, with muscle contractions without change in their length according to verbal and tactile stimulation given by the therapist according to the need presented by the volunteer.

Statistical analysis was performed in the BioEstat 5.3 program, where descriptive statistics were used to characterize the sample, with a significance level of $p \leq 0.05$ for the null hypothesis. Initially, to test the assumptions of the statistical tests, the D'Agostino normality test was applied to the respiratory force variables. The Bonferroni test was used for analysis of variance of maximal inspiratory force and maximal expiratory force, for pre and post-treatment comparisons and for Student's t-test for QoL.

\section{RESULTS}

The sample of this study was composed, in its totality, of $100 \%$ male subjects, the average age of $48.8 \pm 6.22$ years, and the initial diagnosis of PD occurred on average $5 \pm 2.91$ years. It was found through the modified Hoehn and Yahr scale, $80 \%$ of patients were in stage 2 and $20 \%$ in stage 3 . In relation to the values predicted for respiratory muscle strength there was a positive increase, the predicted MIP values $(-109.5 \pm 7.8)$ reached an average of $-150.7 \pm 76.4$ after treatment $(p<0.05)$. Regarding MEP, statistical differences in post-treatment of predicted value were identified $118.9 \pm 7.9$ compared to pre-treatment $(P<0.05)$, as well as when the predicted value in the post-treatment GPR treatment $86.7 \pm 23.5$ ) $(p<0,05)$. When comparing the averages of the volunteers in the pre- and post-treatment of GPR, the initial MIP was $-100.34 \pm 55.7$, resulting in $-150.7 \pm 76.4$ at the end of the treatment, revealing statistical significance $(p<0.05)$. For MEP, with values of $85.33 \pm 18.8$ pre-treatment and $86.7 \pm 23.5$ post-treatment, the difference did not reach statistical significance ( $p>0.05)$. In regard to the analysis of the QoL, on table 1 shows there was statistically significant improvement for the studied sample in the domains: mobility $(p=0.0009)$, daily life activity $(p=0.0006)$, emotional well-being $(p=0.001)$, cognition $(p=0.01)$, physical discomfort $(p=0.0007)$ and total score $(p=0.0001)$.

\section{DISCUSSION}

PD is the second most prevalent neurodegenerative disease in the elderly because such motor symptoms (tremor, bradykinesia, rigidity and postural instability) and non-motor (neuropsychiatric, sensorial and autonomic disorders and sleep disorders) affect the individual's life in a multidimensional way with functional limitation, causing greater dependence
Table 1. Intragroup comparisons of PDQ-39 domains before and after Global Postural Reeducation treatment.

\begin{tabular}{lccl}
\hline \multicolumn{1}{c}{ Domains } & Pre-treatment & Post-treatment & P value \\
\hline Mobility & $11.3 \pm 6.8$ & $4.0 \pm 3.3$ & $0.0009^{*}$ \\
$\begin{array}{l}\text { Daily living } \\
\text { activity }\end{array}$ & $8.5 \pm 5.1$ & $3.0 \pm 2.4$ & $0.0006^{*}$ \\
$\begin{array}{l}\text { Emotional well- } \\
\text { being }\end{array}$ & $9.3 \pm 4.9$ & $4.1 \pm 3.6$ & $0.001^{*}$ \\
$\begin{array}{l}\text { Stigma } \\
\text { Social support }\end{array}$ & $2.4 \pm 2.3$ & $1.1 \pm 1.1$ & 0.2 \\
Cognition & $0.7 \pm 1.3$ & $0.6 \pm 1.2$ & 0.8 \\
Communication & $3.4 \pm 2.5$ & $1.1 \pm 0.9$ & $0.01^{*}$ \\
$\begin{array}{l}\text { Physical } \\
\text { discomfort }\end{array}$ & $3.3 \pm 1.0$ & $3.3 \pm 1.0$ & 1.0 \\
Total Score & $6.4 \pm 1.1$ & $2.9 \pm 1.5$ & $0.0051^{*}$ \\
\hline
\end{tabular}

*statistical significance

on ADL's and lower QoL ${ }^{(15)}$. CNS involvement culminates in chronic degeneration of the dopaminergic neurons located in the compact part of the neuronal black substance and causes difficulties in motor control and impairment of respiratory function, due to loss of flexibility of the respiratory muscles, postural alterations, alterations in muscle activation and coordination $^{(16)}$. The patients in the sample between levels 2 and 3 on the modified Hoehn and Yahr Scale presented moderate impairment, with marked motor alterations and manifestation of parkinsonian tetrad.

Although, much of the study of PD motor complications and correlating respiratory dysfunctions in this context, few studies describe this condition ${ }^{(17)}$. The adoption of GPR postures increase the flexibility, expandability and strength of the respiratory muscles, as regards MIP $(p<0.05)$, in which the final mean $(-150.7 \pm 76.4)$ exceeding the initial value $(-100.34 \pm 55.7)$. This occurs, physiologically, by stimulating addition of sarcomeres serially and in parallel, actively during the execution of the stretching positions of the shortened inspiratory musculature ${ }^{(18)}$. However, the final mean MEP was $86.7 \pm 23.5$, not statistically significant difference $(p>0.05)$ with the pretreatment value of $85.33 \pm 18$.8. Patients with PD tend to present worsening of pulmonary function mainly due to postural alteration, and this worsening may increase with disease progression ${ }^{(19)}$. In our study, perhaps, the disease time and degree of the disease did not contribute to the reduction of expiratory muscle strength. Other studies prove the efficacy of GPR as a beneficial treatment on ventilatory biomechanics by referring to the individual as a whole and not the disease, since the changes involve all systems. Through their postures, stretching, body recognition, flexibility, contractions and relaxation, the respiratory muscle chains are reeducated, returning their correct functionalities, improving body mobility, postural correction, tonicity, strengthening, rebalancing, body awareness and harmonization of the chains ${ }^{(18,20)}$. 
A prospective study of 20 male students of the Physiotherapy course at the Methodist University of Piracicaba corroborates these findings, were a group was submitted to the relaxation maneuver of the diaphragm at the beginning, preparing it for stretching. They used GPR's "frog on the floor with open arms" posture to stretch the respiratory muscle strings for 30 minutes, twice a week for eight weeks, totaling 16 sessions. At the end we observed that the treated group obtained a significant difference to the spirometric data collected before the training ${ }^{(21)}$. During the performance of the GPR technique, there are cardiovascular alterations it may be justified by thoracic mobility, it was observed in a study done with 09 university students, showing an increase in physiological variables such as systolic blood pressure, diastolic blood pressure, mean arterial pressure and double product in healthy individuals, it may also have occurred in the present study, but it was not investigated, however, according to the authors, physiological values return immediately to the end of the technique by thoracic re-adjustment, this alteration of the thoracic cavity may increase the work respiratory rate, it culminates in an increase in the values of respiratory muscle strength throughout the protocol applied ${ }^{(22)}$.

Studies on GPR efficacy are scarce, even though it is widely used in clinical practice. The technique already comprises the treatment of dysfunctions / pathologies such as stroke, temporomandibular dysfunction, scoliosis, fibromyalgia, urinary incontinence, among others ${ }^{(23)}$. Though it is still difficult to find literatures portraying GPR in the individual with PD. The PD patient usually assumes a characteristic posture with the head in slight flexion, trunk leaning forward, moderate flexion of the leg over the thigh and the forearm over the arm, ventrally flexed trunk and digital tweezers in the hands, as a compensatory posture and safety due to motor alterations and difficulty in anticipatory and balance reactions ${ }^{(24)}$. Depression and motor and non-motor aspects in ADL's directly affect patients' independence, which may justify a reduction in the QoL of patients with PD ${ }^{(25)}$. The subjects with PD has impaired functional capacity, evolving over time. In addition to the CNS changes, the patient still suffers from the functional decline due to aging, it generally leads to their social isolation, physical inactivity, fragility, risk of falls, mobility problems, among others, and consequently result in dependence and poor QoL ${ }^{(26)}$

The present study demonstrated GPR postures promote a statistically significant improvement in QoL in the domains of mobility ( $p=0.0009)$, ADL's $(p=0.0006)$, well being emotional $(p=0.001)$, cognition ( $p=0.01)$, physical discomfort $(p=0.0007)$ and total score $(p=0.0001)$. GPR generates benefits in the parkinsonian quality of life, due to the personal and quantitative unblocking and reduction of its functional limitations, through proprioceptive stimulation, tonus, strengthening, flexibility and stretching ${ }^{(27)}$.
The Pilates method (PM) has similar benefits to GPR, as both favor balance and motor coordination, functional independence, body awareness, postural correction, increase muscle strength and endurance, decrease shortening, improve flexibility, physical fitness, mental, respiratory capacity, ADL, self-esteem and $\mathrm{QOL}^{(20,28)}$. In the case study, a participant, 67 years of age was diagnosed with PD, a protocol was elaborated with 20 PM exercises in soil, ball and apparatus (Cadillac, Step Chair and Reformer). As a result, PDQ-39 $9^{(13)}$ showed an improvement of $76.8 \%$ in the QoL of the volunteer ${ }^{(28)}$. The experimental study of an uncontrolled clinical trial, performed at the neurology outpatient clinic of the Hospital das Clínicas of the Federal University of Pernambuco, also measured QoL through PDQ-39 and verified improvement in several domains of the questionnaire, including cognition, after the virtual reality ${ }^{(11)}$.

\section{CONCLUSION}

The present study contributes with significant results adopting GPR postures method, it helped to increase inspiratory muscle strength and the quality of life of patients with Parkinson's disease.

\section{AUTHOR'S CONTRIBUTION}

LSO: conception and design of this study; LNHM: data acquisition, draft and revision of the study; LLS: analysis and interpretation of data, draft and revision of the study; LDM: data acquisition, draft and revision of the study; MCSS: analysis and interpretation of data; RSBR: conception and design of this study, final approval of the version to submission to publication.

\section{CONFLICTS OF INTEREST}

Nothing to declare

\section{AUTHOR DETAILS}

1 Centro Universitário do Estado do Pará (CESUPA), Belém (PA), Brazil.

${ }^{3}$ Universidade da Amazônia (UNAMA), Belém (PA), Brazil.

\section{REFERENCES}

1. Rodrigues AV, Lemes LB, Rodrigues ASR, Souza RB, Santos SMS. Evaluation of the Perceptual System in Healthy Elderly and Elderly Patients with Parkinson's Disease. Rev Neurocienc. 2014;22(2):189-194

2. Curtze C, Nutt JG, Carlson-Kuhta P, Mancini M, Horak FB. Objective Gait and Balance Impairments Relate to Balance Confidence and Perceived Mobility in People with Parkinson Disease. Phys Ther. 2016;96(11):17341743.

3. Blaszczyk JW. Nigrostriatal interaction in the aging brain: new therapeutic target for Parkinson's disease. Acta Neurobiol Exp (Wars). 2017;77(1):106112.

4. Silva FP, Martinello M, Medeiros DL, Schwertnerd DS, Ries LGK. Global postural reeducation in an adult with cerebral palsy: a case study. Fisioter Pesq. 2015;22(1):90-96.

5. Tasca C, Schuster RC, Alvarenga LFC. Respiratory muscle strength and thoracic mobility in patients with Parkinson's disease. Journal of Health Care. 2014;12(42):5-10.

6. Bertoldi FC, Silva JA, Faganello-Navega FR. Influence of muscle strengthening on balance and quality of life in individuals with Parkinson's disease. Fisioter. Pesqui. 2013;20(2):117-122. 
7. Gomes AVM, Lopes DC, Veloso EMC, Costa RCTS. The influence of the global postural reeducation method on the flexibility of the posterior chain muscles. Fisioter Bras. 2014;15(3):200-206.

8. Tavares GMS, Santo CCE, Parizotto P, Sperandio FF, Santos GM. Treatment for scoliosis by the global postural reeducation method (GPR) in the total visually impaired: series of cases. Sci Med. 2015;25(3):1-8.

9. Teodori RM; Negri JR; Cross MC; Marques AM. Global Postural Reeducation: a review of the literature. Rev Bras Fisioter. 2011;15(3):185189.

10. Buated W, Lolekha P, Hidaka S, Fujinami T. Impact of cognitive loading on postural control in Parkinson's disease with freezing of gait. Geront Geriat Med. 2016;2(1):1-8.

11. Christofoletti G, Oliani MM, Gobbi LTB, Gobbi S, Stella F. Risk of falls in the elderly with Parkinson's disease and Alzheimer's dementia: a crosssectional study. Rev Bras Fisioter. 2006;10(4):429-433.

12. Battaglia E, Fulgenzi A, Ferrero ME. Rationale of the combined use of inspiratory and expiratory devices in improving maximal inspiratory pressure and maximal expiratory pressure of patients with chronic obstructive pulmonary disease. Arch Phys Med Rehabil. 2009;90(6):913918.

13. Santana CMF, Lins OG, Sanguinetti DCM, Silva FP, Angelo TDA, Coriolan MGWS. Effects of non-immersive virtual reality treatment on the quality of life of individuals with Parkinson's. Rev. Bras. Geriatr. Gerontol. 2015;18(1):49-58.

14. Oliveri M, Caltagirone C, Loriga R, Pompa MN, Versace V, Souchard P. Fast increase of motor cortical inhibition following postural changes in healthy subjects. Neurosci Lett. 2012;530(1):7-11.

15. Pinheiro IM, Santos LLS, Paula LCN, Costa ACN. Impact of Parkinson 's disease on the functionality and quality of life of the elderly in a geriatric reference unit in the city of Salvador - Bahia. Rev. Ciênc. Med. Biol. 2014;13(3):292-297.

16. Baptista A, Vieira RTB, Silva SM, Correa JCF, Sampaio LM, Correa FI. Immediate effect of thoracoabdominal rebalancing on the respiratory mechanics of parkinsonians. Fisioter Bras. 2014;15(2):131-135.

17. Wang CM, Shieh WY, Weng YH, Hsu YH, Wu YR. Non-invasive assessment of swallowing and respiratory dysfunction in early Parkinson's disease. Parkinsonism Relat Disord. 2017;S1353-8020(17):30190-6.
18. Guimarães EA, Alkmim DN, Birth MC, Boaventura CM, Magazoni VS. Adaptations of the respiratory system related to pulmonary function in response to a muscular treatment program by the Global Posture Reeducation method. ConScientiae Saúde. 2013;12(1):97-105.

19. Hampson NB, Kieburtz KD, Lewitt PA, Leinonen M, Freed MI. Prospective evaluation of pulmonary function in Parkinson's disease patients with motor fluctuations. Int J Neurosci. 2017;127(3):276-284.

20. Comerlato T, Scanegatta S, Rosset D. Effects of the Global Posture Reeducation (GPR) method in the treatment of scheuermann kyphosis. FisiSenectus. 2013;1:10-19.

21. Moreno MA, Catai AM, Teodori RM, Borges BLA, Zuttin RS, Silva E. Respiratory system adaptations regarding pulmonary function in response to a muscle stretching program using the Global Posture Reeducation method. Fisioter Pesq. 2009;16(1):11-15.

22. Mota YL, Barreto SL, Bin PR, Simões HG, Campbell CSG. Cardiovascular responses during the posture of Global Posture Reeducation (GPR). Rev Bras Fisioter. 2008;12(3):161-168.

23. Refice C, Loriga R, Pompa MN, Gambale G, Altavilla R, Paolucci M et al. Global postural rehabilitation and migraine: a pilot-study. J Headache Pain. 2015;16(Suppl 1):A116.

24. Lira TBS, Barros ALS, Costa RV, Lustosa TC, Silva SACD. Analysis of the posture of subjects with stage II Parkinson's disease. R. Ci. Med. Biol. 2012;11(3):296-300.

25. Kadastik-Eerme L, Rosenthal M, Paju T, Muldmaa M, Taba P. 2015). Health-related quality of life in Parkinson's disease: a cross-sectional study focusing on non-motor symptoms. Health What Life Outcomes. 2015;13(83):1-8.

26. Fleck CS, Gerzson LR, Steidl EMS, Hernandez NM. Characterization of functional capacity, cognitive level and respiratory muscle strength of elderly women with parkinsonian syndrome. Estud. interdiscipl. Envelhec. 2014;19(1):109-121.

27. Silva AO, Oliveira FL, Alves AG, Nogueira MS, Valente PHF, Souza EL, et al. Effect of the global postural reeducation technique (GPR) in the treatment of patients with lumbar hyperlordosis: a case study. Revista Eletrônica FMB 2016;9(1):102-173.

28. Freitas MLM, Zager M, Campbell C. The influence of the Pilates method on the postural instability and quality of life of patients with Parkinson's disease. Fisioter Bras. 2015;16(2):155-159. 